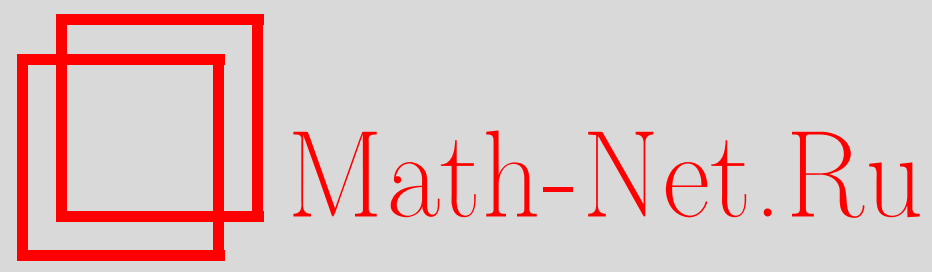

А. А. Саакян, Сходимость двойных рядов Фурье после замены переменной, Матем. заметки, 2003, том 74, выпуск 2, 267-277

DOI: https://doi.org/10.4213/mzm263

Использование Общероссийского математического портала Math-Net.Ru подразумевает, что вы прочитали и согласны с пользовательским соглашением http://www . mathnet.ru/rus/agreement

Параметры загрузки:

IP : 54.92 .164 .108

26 апреля 2023 г., $17: 17: 01$ 
УДК 517.518

\section{СХОДИМОСТЬ ДВОЙНЫХ РЯДОВ ФУРЬЕ ПОСЛЕ ЗАМЕНЫ ПЕРЕМЕННОЙ}

\section{А. А. Саакян}

В работе доказьвается, что для любого компакта $\Omega \subset C\left(\mathbb{T}^{2}\right)$ существует гомеоморфизм $\tau$ отрезка $\mathbb{T}=[-\pi, \pi]$ такой, что для произвольной функции $f \in \Omega$ ряд Фурье функции $F(x, y)=f(\tau(x), \tau(y))$ равномерно сходится на $C\left(\mathbb{T}^{2}\right)$ одновременно и по прямоугольикам, и по сферам, и по треугольникам.

Библиография: 10 названий.

1. Введение. Пусть $C(\mathbb{T})$ (соответственно $C\left(\mathbb{T}^{2}\right)$ ) - пространство непрерывных, $2 \pi$-периодических (по каждой переменной) функций на $\mathbb{R}^{1}$ (соответственно $\mathbb{R}^{2}$ ). Для одномерных тригонометрических рядов Фурье хорошо известна (см. [1, с. 303])

ТЕорема А (Бор). Для $f \in C(\mathbb{T})$ существует гомеоморфизм $\tau(t)$ отрезка $\mathbb{T}=$ $[-\pi, \pi]$, т.е. непрерывная функиия с условием

$$
-\pi=\tau(-\pi)<\tau\left(t_{1}\right)<\tau\left(t_{2}\right)<\tau(\pi)=\pi, \quad \pi<t_{1}<t_{2}<\pi
$$

такая, что ряд Фурье суперпозичии $f$ о $\tau(t)$ равномерно сходится на $\mathbb{T}$.

В работах [2], [3] были получены разные усиления этой теоремы. В частности, было доказано, что гомеоморфизм $\tau$ в теореме Бора можно построить единьп для заданного компакта в $C(\mathbb{T})$. А именно, справедлива

Теорема В (Кахан, Кацнельсон [2]). Пусть

$$
\omega \in C(0, \infty), \quad 0=\omega(0)<\omega\left(\delta_{1}\right)<\omega\left(\delta_{2}\right)<\infty, \quad 0<\delta_{1}<\delta_{2}<\infty
$$

Существует гомеоморфизм $\tau$ отрезка $\mathbb{T}$ такой, что для произвольной функиии $f \in C(\mathbb{T})$ с модулем непрерывности $\omega(\delta, f) \leqslant \omega(\delta)$ ряд Фурье суперпозииии $f \circ \tau(t)$ равномерно сходится на $\mathbb{T}$.

Пусть $S_{n, m}(F, x, y)$ - прямоугольные частные суммы ряда $\Phi$ урье интегрируемой на $\mathbb{T}^{2}$ функции $F$ :

$$
S_{n, m}(F, x, y)=\sum_{k=-n}^{n} \sum_{j=-m}^{m} c_{k, j}(F) e^{i(k x+j y)}, \quad(x, y) \in \mathbb{T}^{2}, \quad n, m=1,2, \ldots,
$$


где $c_{k, j}(F)$ - коэффициенты Фурье функции $F$ :

$$
c_{k, j}(F)=\frac{1}{2 \pi} \int_{-\pi}^{\pi} \int_{-\pi}^{\pi} F(x, y) e^{-i(k x+j y)} d x d y, \quad k, j=0, \pm 1, \pm 2, \ldots
$$

Напомним, что сходимость ряда Фурье функции $F$ по прямоугольникам (по Прингсхеймy) означает сходимость прямоугольных частных сумм при $n, m \rightarrow \infty$.

Для $F \in C\left(\mathbb{T}^{2}\right)$ обозначим

$$
\omega(\delta, F)=\sup _{\left(x_{1}-x_{2}\right)^{2}+\left(y_{1}-y_{2}\right)^{2} \leqslant \delta^{2}}\left|F\left(x_{1}, y_{1}\right)-F\left(x_{2}, y_{2}\right)\right|, \quad 0<\delta<\infty .
$$

Теорема Кахана и Кацнельсона была обобщена нами на двумерньй случай для сходимости по Прингсхейму:

Теорема С (Саакян [4]). Для произвольной функиии $\omega(\delta)$ с условиями (1.2) существует гомеоморфизм $\tau(t)$ отрезка $T$ такой, что ряд Фурье произвольной функиии F вида

$$
F(x, y)=f(\tau(x), \tau(y)), \quad f \in C\left(\mathbb{T}^{2}\right), \quad \omega(\delta, f) \leqslant \omega(\delta)
$$

равномерно сходится по Прингсхейму на квадрате $\mathbb{T}^{2}$.

Более того, для произвольного в >0 существует номер $N$ такой, что

$$
\left\|S_{n, m}(F)-F\right\|_{C} \leqslant \varepsilon \quad n p u \quad n, m>N
$$

для всех функиий $F$ вида (1.3).

Как известно, сходимость кратных рядов можно определить разными способами. В настоящей работе мы изучим возможность распространения этой теоремы на другие способы сходимости. Для двойного числового ряда

$$
\sum_{k, j=-\infty}^{\infty} a_{k, j}
$$

положим

$$
\begin{gathered}
S_{n, m}=\sum_{k=-n}^{n} \sum_{j=-m}^{m} a_{k, j}, \quad n, m=0,1 \ldots, \\
S_{R}=\sum_{k^{2}+j^{2} \leqslant R^{2}} a_{k, j}, \quad R \geqslant 0, \\
S_{n}^{t}=\sum_{|k|+|j| \leqslant n} a_{k, j}, \quad n=0,1 \ldots
\end{gathered}
$$

Если существует конечньй предел

$$
\lim _{n, m \rightarrow \infty} S_{n, m}=a, \quad \lim _{n \rightarrow \infty} S_{n, n}=a, \quad \lim _{R \rightarrow \infty} S_{R}=a, \quad \lim _{n \rightarrow \infty} S_{n}^{t}=a,
$$

тоговорят, что ряд (1.5) сходится к а по прямоугольникам (по Прингсхейму), по квадратам, по сферам, по треугольникам соответственно.

В работах Арутюняна [5] и Дьяченко [6] были введены понятия $u$-сходимости и $u(K)$-сходимости кратных рядов. 
ОПРЕДЕЛЕНИЕ 1. Говорят, что конечное множество $U \subset \mathbb{Z}^{2}$ принадлежит клас$c y A_{3}$, если вместе с произвольной точкой $(k, j) \in U$ множеству $U$ принадлежат также все целочисленные точки прямоугольника

$$
[-|k|,|k|] \times[-|j|,|j|]
$$

Если, кроме того, при фиксированном $K>1$ из условий $(0, N) \in U,(M, 0) \in U$ следует, что множества

$$
\left\{(k, j) \in \mathbb{Z}^{2}: K|k|+|j| \leqslant N\right\}, \quad\left\{(k, j) \in \mathbb{Z}^{2}:|k|+K|j| \leqslant M\right\}
$$

лежат в $U$, то говорят, что $U$ принадлежит классу $A_{3}(K)$.

Заметим, что если $U \in u(K)$, то $N(U) / n(U) \leqslant 2 K$, где

$$
\begin{aligned}
N(U) & :=\min \left\{n:[-n, n]^{2} \cap \mathbb{Z}^{2} \supseteq U\right\}, \\
n(U) & :=\max \left\{n:[-n, n]^{2} \cap \mathbb{Z}^{2} \subseteq U\right\} .
\end{aligned}
$$

ОПРЕДЕЛЕНИЕ 2. Говорят, что ряд (1.5) $u$-сходumcя (соответственно $u(K)$ - $c x о д u m$ ся) к $a$, если для любого $\varepsilon>0$ существует число $N$ такое, что

$$
\left|\sum_{(k, j) \in U} a_{k, j}-a\right|<\varepsilon
$$

для любого $U \in A_{3}$ (соответственно $\left.U \in A_{3}(K)\right)$ с $n(U)>N$.

Ясно, что из $u(K)$-сходимости ряда следует ее сходимость и по квадратам, и по сферам, и по треугольникам, но не следует сходимость по Прингсхейму.

Для конечного множества $U \subset \mathbb{Z}^{2}$ обозначим

$$
S_{U}(F, x, y)=\sum_{(k, j) \in U} c_{k, j}(F) e^{i(k x+j y)} .
$$

Основным результатом статьи является следующая

Теорема 1. Для произвольной функиии $\omega(\delta)$ с условиями (1.2) существует гомеоморфизм $\tau(t)$ отрезка $\mathbb{T}$ такой, что ряд Фурье произвольной функиии $F$ вида (1.3) $и(K)$-сходится для любого $K>1$ и сходится по Прингсхейму равномерно на квадрате $\mathbb{T}^{2}$.

Более того, для произвольных $\varepsilon>0 и K>1$ существует номер $N$ такой, что имеет место (1.4) и

$$
\left\|S_{U}(F)-F\right\|_{C} \leqslant \varepsilon \quad n p u \quad n(U)>N, \quad U \in A_{3}(K),
$$

для всех функиий $F$ вида (1.3).

Нам понадобится ряд обозначений. Для функции $f \in C(\mathbb{T})$ и интервала $\Delta=(a, b)$ обозначим

$$
f(\Delta)=f(b)-f(a), \quad f^{*}(\Delta)=\sup _{a \leqslant x<y \leqslant b}|f(x)-f(y)| .
$$


Обозначим также

$$
\Delta_{k}^{1}(x, h)=(x+(k-1) h, x+k h), \quad \Delta_{k}^{-1}(x, h)=(x-k h, x-(k-1) h),
$$

где $x \in \mathbb{T}, h \in(0, \pi), k=1,2, \ldots$

Рассмотрим теперь произвольную последовательность $\Lambda=\left\{\lambda_{k}\right\}_{k=1}^{\infty}$ с условиями

$$
1 \leqslant \lambda_{1} \leqslant \lambda_{2} \leqslant \cdots, \quad \sum_{k=1}^{\infty} \frac{1}{\lambda_{k}}=\infty
$$

и обозначим

$$
\begin{aligned}
\Lambda V_{h}^{\gamma}(f, x) & :=\sum_{k=1}^{[\pi / h]} \frac{f^{*}\left(\Delta_{k}^{\gamma}(x, h)\right)}{\lambda_{k}}, \quad x \in \mathbb{T}, \quad h \in(0, \pi), \quad \gamma= \pm 1, \\
\Lambda V_{h}(f): & =\sup _{x \in T, \gamma= \pm 1} \Lambda V_{h}^{\gamma}(f, x), \quad h \in(0, \pi) .
\end{aligned}
$$

Пусть теперь $F(x, y) \in C\left(\mathbb{T}^{2}\right)$. Для интервалов $\Delta_{1}=\left(a_{1}, a_{2}\right)$ и $\Delta_{2}=\left(b_{1}, b_{2}\right)$ положим

$$
\begin{aligned}
F\left(\Delta_{1}, \Delta_{2}\right) & :=F\left(a_{1}, b_{1}\right)+F\left(a_{2}, b_{2}\right)-F\left(a_{1}, b_{2}\right)-F\left(a_{2}, b_{1}\right), \\
F^{*}\left(\Delta_{1}, \Delta_{2}\right) & :=\sup |F(I, J)|,
\end{aligned}
$$

где sup берется по всем интервалам $I \subset \Delta_{1}$ и $J \subset \Delta_{2}$.

Для $(x, y) \in \mathbb{T}^{2}, h \in(0, \pi), \gamma_{1}, \gamma_{2}= \pm 1$ определим величину

$$
\Lambda V_{h}^{\gamma_{1}, \gamma_{2}}(F, x, y):=\sum_{k, j=1}^{[\pi / h]} \frac{F^{*}\left(\Delta_{k}^{\gamma_{1}}(x, h), \Delta_{j}^{\gamma_{2}}(y, h)\right)}{\lambda_{k} \lambda_{j}}
$$

и положим

$$
\Lambda V_{h}(F):=\sup \Lambda V_{h}^{\gamma_{1}, \gamma_{2}}(F, x, y)+\sup \Lambda V_{h}^{\gamma_{1}}(F(\cdot, y), x)+\sup \Lambda V_{h}^{\gamma_{2}}(F(x, \cdot), y)
$$

где $\sup$ берется по $(x, y) \in \mathbb{T}^{2}, \gamma_{1}, \gamma_{2}= \pm 1$.

Всюду ниже через $C$ мы обозначаем абсолютные константы, которые могут быть разные в разных формулах.

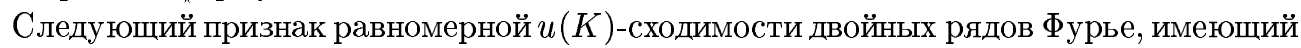
самостоятельньй интерес, играет важную роль в доказательстве теоремы 1.

Tеорема 2. Пусть $\lambda_{k}=\sqrt{k} / \sqrt{\ln (k+1)}, k=1,2, \ldots$ Ecлu $F \in C\left(\mathbb{T}^{2}\right) u$

$$
\lim _{h \rightarrow 0} \Lambda V_{h}(F)=0
$$

то ряд Фурье функиии F равномерно $и(K)$-сходится на $\mathbb{T}^{2}$ для любого $K>1$.

Более того, имеет место оценка

$$
\left\|S_{U}(F)-F\right\|_{C} \leqslant C K\left\{\Lambda V_{h(U)}(F)+\omega(h(U), F)\right\}, \quad h(U)=\frac{\pi}{n(U)},
$$

для любого множества $U \in u(K), K>1$. 
ЗАмЕчАниЕ 1. Этот признак можно считать некоторьм аналогом хорошо известного признака Салема. Для двойных рядов аналог признака Салема для равномерной сходимости по Прингсхейму был доказан Голубовым в [7]. Для прямоугольных частных сумм оценка, аналогичная (1.11) (с $\left.\lambda_{k} \equiv k\right)$, была доказана нами в [4].

Легко видеть, что $\omega(h, F) \rightarrow 0$ при $h \rightarrow 0$ равномерно по множеству функций $F$ вида (1.3). Это означает, что теорема 1 вытекает из теоремы 2 и из следующей леммы (см. также замечание 2).

Лемма 1. Для произвольной последовательности $\Lambda=\left\{\lambda_{k}\right\}_{k=1}^{\infty}$ вида (1.8) $u$ любой функции $\omega(\delta)$ вида (1.2) существует гомеоморфизм $\tau(t)$ отрезка $\mathbb{T}$ такой, что имеет место (1.10) равномерно по множсеству функиий $F$ вида (1.3).

ЗАмЕчАниЕ 2. Аналогичная лемма была доказана нами для последовательности $\lambda_{k} \equiv k$ и для величины $V_{h}(F)$, которая соответствует прямоугольным частичньм суммам ряда Фурье (см. подробнее [4, лемма 5]). Нетрудно убедиться, что гомеоморфизм $\tau$, построенный в доказательстве леммы 1 , удовлетворяет также требованиям леммы 5 из работы [4].

Отметим, что величина $\Lambda V_{h}(F)$ имеет много общего с так назьваемой $\Lambda$-вариацией функции $F$, которая определяется следующим образом:

$$
\Lambda V(F)=\sup \sum_{k, j} \frac{\left|F\left(I_{k}, \Delta_{j}\right)\right|}{\lambda_{k} \lambda_{j}}+\sup \sum_{k} \frac{\left|F\left(I_{k}, 0\right)\right|}{\lambda_{k}}+\sup \sum_{j} \frac{\left|F\left(0, \Delta_{j}\right)\right|}{\lambda_{j}},
$$

где sup берется по всевозможным парам систем $\left\{I_{k}\right\}$ и $\left\{\Delta_{j}\right\}$ непересекающихся интервалов из $\mathbb{T}$. Класс измеримых на $\mathbb{T}^{2}$ функций $F$ с ограниченной $\Lambda$-вариацией обозначают

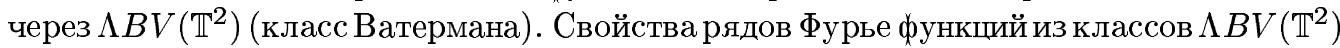
изучались во многих работах. Было доказано, в частности, что ряд Фурье функции $F \in C\left(\mathbb{T}^{2}\right):$

1) (Саакян [8]) равномерно сходится по Прингсхейму, если $F \in \Lambda B V\left(\mathbb{T}^{2}\right)$ с $\lambda_{k} \equiv k$,

2) (Дьяченко [9]) равномерно сходится по сферам, если $F \in \Lambda B V\left(T^{2}\right)$ с $\lambda_{k}=o(\sqrt{k})$,

3) (Дьяченко [6]) равномерно $u(K)$-сходится для любого $K>1$, если $F \in \Lambda B V\left(T^{2}\right)$ с

$$
\lambda_{k}=o\left(\frac{\sqrt{k}}{\sqrt{\ln (k+1)}}\right) \text {. }
$$

ЗАмЕчАнИЕ 3. Нетрудно проверить, что если последовательность $\Lambda=\left\{\lambda_{k}\right\}$ удовлетворяет условию (1.12), то из $F \in C\left(\mathbb{T}^{2}\right) \cap \Lambda B V\left(\mathbb{T}^{2}\right)$ следует, что $F$ удовлетворяет условиям теоремы 2 . Это означает, что теорема 2 усиливает вышеупомянутьй результат Дьяченко (см. 3)). Для нас это усиление существенно, так как для доказательства теоремы 1 мы не можем воспользоваться результатом Дьяченко вместо теоремы 2: очевидно, что $\Lambda$-вариация функции инвариантна относительно гомеоморфизмов вида (1.3), и в лемме 1 условие (1.10) нельзя заменить на ограниченность $\Lambda$-вариации функции $F$.

В то же время следует отметить, что оценки и рассуждения, необходимые для получения (1.11), в основном содержатся в работе [6].

ЗАмЕчАниЕ 4. Если в приведенном ниже доказательстве теоремы 2 вместо оценок (2.2)-(2.5) воспользоваться соответствующими оценками для сферических ядер, полученньми Дьяченко в работе [9] (см. лемму 5 и следствия 2 и 3 ), можно доказать, что для сходимости по сферам теорема 2 верна с $\lambda_{k} \equiv \sqrt{k}$. 
ЗАмечАниЕ 5 . Теорема 2 перестает быть верной для последовательности вида $\Lambda_{\alpha}=\left\{\lambda_{k}=\sqrt{k}(\ln (k+1))^{\alpha}\right\}$ при $\alpha>0$. Это вытекает из одного результата Бахвалова, согласно которому для любого $\alpha>0$ сушествует функция $F \in \Lambda_{\alpha} B V\left(\mathbb{T}^{2}\right)$, треугольные частные суммы которой не ограничены равномерно (см. [10]).

2. Доказательства. Для произвольного конечного множества $U \subset \mathbb{Z}^{2}$ и интегрируемой функции $F$ имеем

$$
S_{U}(F, x)=\frac{1}{\pi^{2}} \int_{T^{2}} F(x+t, y+s) D_{U}(t, s) d t d s,
$$

где $D_{U}(t, s)$ - ядро Дирихле:

$$
D_{U}(t, s)=\sum_{(m, n) \in U} e^{-i(m t+n s)}
$$

Лемма 2 (Дьяченко [6]). Для любого множества $U \in A_{3}(K), K>1$, и для всех $x, y \in[0, \pi]$ справедливы следующие оченки:

$$
\begin{aligned}
\left|D_{U}(x, y)\right| & \leqslant C K \frac{n^{2}(U)}{\sqrt{n(U) x+1} \sqrt{n(U) y+1}}, \\
\left|\int_{x}^{\pi} D_{U}(t, y) d t\right| & \leqslant C K \frac{n(U) \sqrt{\ln (n(U) x+2)} \sqrt{\ln (n(U) y+2)}}{\sqrt{n(U) x+1} \sqrt{n(U) y+1}}, \\
\left|\int_{y}^{\pi} D_{U}(x, s) d s\right| & \leqslant C K \frac{n(U) \sqrt{\ln (n(U) x+2)} \sqrt{\ln (n(U) y+2)}}{\sqrt{n(U) x+1} \sqrt{n(U) y+1}}, \\
\left|\int_{x}^{\pi} \int_{y}^{\pi} D_{U}(t, s) d t d s\right| & \leqslant C K \frac{\sqrt{\ln (n(U) x+2)} \sqrt{\ln (n(U) y+2)}}{\sqrt{n(U) x+1} \sqrt{n(U) y+1}} .
\end{aligned}
$$

ДоКАЗАТЕЛЬСТво теоРемЫ 2. Достаточно доказать, что существует абсолютная постоянная $C$ такая, что для любого множества $U \in u(K)$ и для произвольной точки $(x, y) \in \mathbb{T}^{2}$ имеет место неравенство

$$
\left|S_{U}(F, x, y)-F(x, y)\right| \leqslant C K\left\{\Lambda V_{h(U)}(F)+\omega(h(U), F)\right\} .
$$

Без ограничения обшности можем считать, что $x=y=0$. Обозначим

$$
S_{U}^{i}(F, 0,0)=\frac{1}{\pi^{2}} \int_{Q_{i}} F(t, s) D_{U}(t, s) d t d s
$$

где $Q_{1}=[0, \pi]^{2}, Q_{2}=[-\pi, 0] \times[0, \pi], Q_{3}=[-\pi, 0]^{2}, Q_{4}=[0, \pi] \times[-\pi, 0]$. Согласно $(2.1)$ имеем

$$
S_{U}(F, 0,0)=\frac{1}{\pi^{2}} \int_{T^{2}} F(t, s) D_{U}(t, s) d t d s=\sum_{i=1}^{4} S_{U}^{i}(F, 0,0) .
$$

Учитьвая, что

$$
\frac{1}{\pi^{2}} \int_{Q_{i}} D_{U}(t, s) d t d s=\frac{1}{4}, \quad i=1,2,3,4
$$


будем иметь

$$
\begin{aligned}
S_{U}(F, 0,0)-F(0,0) & =\sum_{i=1}^{4}\left[S_{U}^{i}(F, 0,0)-\frac{1}{4} F(0,0)\right] \\
& =\sum_{i=1}^{4} \frac{1}{\pi^{2}} \int_{Q_{i}}[F(t, s)-F(0,0)] D_{U}(t, s) d t d s \\
& =: \sum_{i=1}^{4} J_{U}^{i}(F) .
\end{aligned}
$$

Оценим величину $J_{U}^{1}(F)$. Пусть $n=n(U)$ и $t_{k}=\pi k / n, k=0,1, \ldots, n$. Рассмотрим интервалы

$$
\Delta_{k}=\left(t_{k-1}, t_{k}\right), \quad \Delta_{k, j}=\Delta_{k} \times \Delta_{j}, \quad k, j=1,2, \ldots, n
$$

и определим функцию

$$
g(x, y)=\sum_{k, j=1}^{n} F\left(x_{k}, y_{j}\right) \chi_{\Delta_{k, j}}(x, y), \quad g(0,0)=F(0,0)
$$

где $x_{k}=y_{k}=t_{k}$, а $\chi_{E}-$ характеристическая функция множества $E$. Сначала мы оценим величину

$$
\begin{aligned}
\pi^{2} J_{U}^{1}(g)= & \sum_{k, j=1}^{n} F\left(t_{k}, s_{j}\right) \int_{\Delta_{k, j}} D_{U}(t, s) d t d s-g(0,0) \int_{0}^{\pi} \int_{0}^{\pi} D_{U}(x, y) d t d s \\
= & \sum_{k, j=2}^{n} F\left(\Delta_{k}, \Delta_{j}\right) \int_{x_{k-1}}^{\pi} \int_{y_{j-1}}^{\pi} D_{U}(t, s) d t d s \\
& +\sum_{k=2}^{n}\left(F\left(x_{k}, y_{1}\right)-F\left(x_{k-1}, y_{1}\right)\right) \int_{0}^{\pi} \int_{x_{k-1}}^{\pi} D_{U}(t, s) d t d s \\
& +\sum_{j=2}^{n}\left(F\left(x_{1}, y_{j}\right)-F\left(x_{1}, y_{j-1}\right)\right) \int_{y_{j-1}}^{\pi} \int_{0}^{\pi} D_{U}(t, s) d t d s \\
& +\left(F\left(x_{1}, y_{1}\right)-F(0,0)\right) \int_{0}^{\pi} \int_{0}^{\pi} D_{U}(t, s) d t d s \\
= & : F_{1}+F_{2}+F_{3}+F_{4} .
\end{aligned}
$$

Применив оценку (2.5), получим

$$
\begin{aligned}
& \left|F_{1}\right| \leqslant C K \sum_{k, j=2}^{n} \frac{F\left(\Delta_{k}, \Delta_{j}\right) \sqrt{\ln (k+1)} \sqrt{\ln (j+1)}}{\sqrt{k} \sqrt{j}} \leqslant C K \cdot \Lambda V_{h(U)}(F), \\
& \left|F_{2}\right| \leqslant C K \sum_{k=2}^{n} \frac{\left|F\left(x_{k}, y_{1}\right)-F\left(x_{k-1}, y_{1}\right)\right| \sqrt{\ln (k+1)}}{\sqrt{k}} \leqslant C K \cdot \Lambda V_{h(U)}(F) .
\end{aligned}
$$


Аналогичным образом находим, что

$$
\left|F_{3}\right| \leqslant C K \cdot \Lambda V_{h}(U)(F),
$$

и наконец,

$$
\left|F_{4}\right| \leqslant C \omega(h(U), F)
$$

Из (2.8)-(2.12) следует, что

$$
J_{U}^{1}(g) \leqslant C K\left\{\Lambda V_{h(U)}(F)+\omega(h(U), F)\right\} .
$$

Теперь мы оценим величину

$$
\begin{aligned}
\pi^{2} J_{U}^{1}(F-g)= & \sum_{k, j=1}^{n} \int_{\Delta_{k, j}}\left(F(t, s)-F\left(x_{k}, y_{j}\right)\right) D_{U}(t, s) d t d s \\
= & \sum_{k, j=1}^{n} \int_{\Delta_{k, j}} F\left(\left(t, x_{k}\right),\left(s, y_{j}\right)\right) D_{U}(t, s) d t d s \\
& +\sum_{k, j=1}^{n} \int_{\Delta_{k, j}}\left(F\left(x_{k}, s\right)-F\left(x_{k}, y_{j}\right)\right) D_{U}(t, s) d t d s \\
& +\sum_{k, j=1}^{n} \int_{\Delta_{k, j}}\left(F\left(t, y_{j}\right)-F\left(x_{k}, y_{j}\right)\right) D_{U}(t, s) d t d s \\
= & G_{1}+G_{2}+G_{3} .
\end{aligned}
$$

Величину $G_{1}$ оценим с помощью $(2.2)$ :

$$
\left|G_{1}\right| \leqslant C K n^{2} \sum_{k, j=1}^{n} \frac{F^{*}\left(\Delta_{k}, \Delta_{j}\right)}{\sqrt{k} \sqrt{j}} \int_{\Delta_{k, j}} d t d s \leqslant C K \cdot \Lambda V_{h(U)}(F) .
$$

Для оценки $G_{2}$ применим преобразование Абеля и воспользуемся неравенством (2.3):

$$
\begin{aligned}
G_{2}= & \sum_{j=1}^{n} \sum_{k=2}^{n} \int_{\Delta_{j}} F\left(\left(x_{k-1}, x_{k}\right),\left(s, y_{j}\right)\right) \int_{x_{k-1}}^{\pi} D_{U}(t, s) d t d s \\
& +\sum_{j=1}^{n} \int_{\Delta_{j}}\left(F\left(x_{1}, s\right)-F\left(x_{1}, y_{j}\right)\right) \int_{0}^{\pi} D_{U}(t, s) d t d s \\
\leqslant & C K n \sum_{j=1}^{n} \sum_{k=2}^{n} \frac{F^{*}\left(\Delta_{k}, \Delta_{j}\right) \sqrt{\ln (k+1)} \sqrt{\ln (j+1)}}{\sqrt{k} \sqrt{j}} \int_{\Delta_{j}} d s \\
& +C K n \sum_{j=1}^{n} F^{*}\left(x_{1}, \Delta_{j}\right) \frac{\sqrt{\ln (j+1)}}{\sqrt{j}} \int_{\Delta_{j}} d s \leqslant C K \cdot \Lambda V_{h(U)}(F) .
\end{aligned}
$$

Аналогичным образом можем убедиться, что $G_{3} \leqslant C K \cdot \Lambda V_{n}(F)$. Отсюда и из $(2.14)-$ (2.16) получим $J_{U}^{1}(F-g) \leqslant C K \cdot \Lambda V_{n}(F)$, что вместе с (2.13) доказьвает неравенство

$$
J_{U}^{i}(F) \leqslant C K\left\{\Lambda V_{h(U)}(F)+\omega(h(U), F)\right\}
$$

при $i=1$. При $i=2,3,4$ оно доказьвается аналогично. Из (2.7) и (2.17) получаем оценку (2.6) для $x=y=0$. Нетрудно проследить, что полученная постоянная $C$ не зависит от выбора точки $(x, y)$ и оценка $(2.7)$ имеет место равномерно по $(x, y) \in \mathbb{T}^{2}$. Теорема 2 доказана. 
ДОКАЗАТЕЛЬСТВО ЛЕММЫ 1 . ИНдукцией по $n=1,2, \ldots$ построим убьвающую последовательность $\left\{\varepsilon_{n}\right\}$ так, чтобы

$$
\varepsilon_{1}=1, \quad \alpha(n):=\frac{\varepsilon_{n-1}}{4 \varepsilon_{n}}>1, \quad \lambda_{\alpha(n)}>16^{n}, \quad n=2,3, \ldots
$$

затем выберем последовательность $\left\{\delta_{n}\right\}$ так, чтобы

$$
0<4 \delta_{n+1}<\delta_{n}<1, \quad \omega\left(\delta_{n}\right) \leqslant 4^{-n} \varepsilon_{n+1}^{2} .
$$

Индукцией по $n=0,1, \ldots$ построим множества

$$
T_{n}=\left\{t_{n}^{i}\right\}_{i=0}^{4^{n}}, \quad A_{n}=\left\{a_{n}^{i}\right\}_{i=0}^{4^{n}} .
$$

Положим $t_{0}^{0}=a_{0}^{0}=-\pi, t_{0}^{1}=a_{0}^{1}=\pi$ и, предположив, что $T_{n-1}$ и $A_{n-1}$ уже построены, положим

$$
\begin{aligned}
& t_{n}^{4 i}=t_{n-1}^{i}, \\
& a_{n}^{4 i}=a_{n-1}^{i}, \\
& i=0, \ldots, 4^{n-1} \text {, } \\
& t_{n}^{4 i-1}=t_{n-1}^{i}-\varepsilon_{n}, \\
& a_{n}^{4 i-1}=\frac{1}{2}\left(a_{n-1}^{i-1}+a_{n-1}^{i}\right)+\delta_{n}, \quad i=1, \ldots, 4^{n-1} \text {, } \\
& t_{n}^{4 i-2}=\frac{1}{2}\left(t_{n-1}^{i-1}+t_{n-1}^{i}\right) \\
& a_{n}^{4 i-2}=\frac{1}{2}\left(a_{n-1}^{i-1}+a_{n-1}^{i}\right), \\
& i=1, \ldots, 4^{n-1}, \\
& t_{n}^{4 i-3}=\frac{1}{2}\left(t_{n-1}^{i-1}+t_{n-1}^{i}\right)-\varepsilon_{n}, \quad a_{n}^{4 i-3}=a_{n-1}^{i-1}+\delta_{n}, \\
& i=1, \ldots, 4^{n-1} \text {. }
\end{aligned}
$$

Легко видеть, что множества $T_{n}$ и $A_{n}$ разбивают отрезок $\mathbb{T}$ на интервалы

$$
d_{n}^{i}=\left(t_{n}^{i-1}, t_{n}^{i}\right), \quad \rho_{n}^{i}=\left(a_{n}^{i-1}, a_{n}^{i}\right), \quad i=1, \ldots, 4^{n},
$$

длины которых удовлетворяют условиям

$$
\begin{gathered}
\left|d_{n}^{2 i}\right|=\varepsilon_{n}, \quad \frac{\varepsilon_{n-1}}{4} \leqslant\left|d_{n}^{2 i-1}\right| \leqslant 2^{-n} \\
\left|\rho_{n}^{2 i}\right| \leqslant 2^{-n}, \quad\left|\rho_{n}^{2 i-1}\right|=\delta_{n}, \quad i=1, \ldots, 4^{n-1 / 2} .
\end{gathered}
$$

Положим

$$
\tau\left(t_{n}^{i}\right)=a_{n}^{i}, \quad i=0, \ldots, 4^{n}, \quad n=0,1, \ldots .
$$

Из (2.20) следует, что функция $\tau$ определена и возрастает на объединении множеств $T_{n}$, которое всюду плотно в $\mathbb{T}$. Так как множество ее значений также всюду плотно в $\mathbb{T}$, мы можем продолжить ее на $\mathbb{T}$ и получить непрерывную функцию вида (1.1). Ясно, что при этом будем иметь

$$
\tau\left(d_{n}^{i}\right)=\rho_{n}^{i}, \quad i=1, \ldots, 4^{n}, \quad n=0,1, \ldots .
$$

Допустим теперь, что $F(x, y)=f(\tau(x), \tau(y)), f \in C\left(\mathbb{T}^{2}\right), \omega(\delta, f) \leqslant \omega(\delta)$, и докажем, что

$$
\lim _{h \rightarrow 0} \Lambda V_{h}(F)=0 .
$$

Фиксируем $h \in(0, \pi)$ и оценим $\Lambda V_{h}(F)$. Выберем $n$ так, чтобы

$$
\varepsilon_{n+1}<h \leqslant \varepsilon_{n} .
$$


Рассмотрим первое слагаемое в (1.9) при $\gamma_{1}=\gamma_{2}=1$ (остальные случаи рассматриваются аналогично):

$$
\Lambda V_{h}^{1,1}(F, x, y)=\sum_{k, j \in \Omega(h)} \frac{\left|F^{*}\left(I_{k}, \Delta_{j}\right)\right|}{\lambda_{k} \lambda_{j}}, \quad I_{k}=\Delta_{k}^{1}(x, h), \quad \Delta_{j}=\Delta_{j}^{1}(y, h),
$$

где

$$
\Omega(h):=\left\{1, \ldots,\left[\frac{\pi}{h}\right]\right\}, \quad \# \Omega(h) \leqslant \frac{\pi}{\varepsilon_{n+1}}
$$

(здесь и в дальнейшем \# $E$ - количество элементов конечного множества $E$ ). Положим

$$
\begin{aligned}
& \Omega_{1}(x)=\left\{k \in \Omega(h): I_{k} \subset d_{n}^{2 i-1} \text { или } I_{k} \subset d_{n+1}^{2 i-1} \text { при некотором } i\right\}, \\
& \Omega_{2}(x)=\left\{k \in \Omega(h) \backslash \Omega_{1}(x): \operatorname{dist}\left(I_{k}, x\right)>\frac{1}{4} \varepsilon_{n-1}\right\}, \\
& \Omega_{3}(x)=\left(\Omega(h) \backslash \Omega_{1}(x)\right) \backslash \Omega_{2}(x) .
\end{aligned}
$$

Аналогичным образом (с $\Delta_{k}$ вместо $I_{k}$ и $y$ вместо $x$ ) определяются множества $\Omega_{1}(y)$, $\Omega_{2}(y), \Omega_{3}(y)$. Из $(2.20)$ и $(2.21)$ следует, что

$$
\left|F^{*}\left(I_{k}, \Delta_{j}\right)\right| \leqslant \begin{cases}2 \omega\left(\delta_{n}\right) & \text { при } k \in \Omega_{1}(x) \text { или } j \in \Omega_{1}(y), \\ 4 \omega\left(2^{-n}\right) & \text { при } k, j \in \Omega(h) .\end{cases}
$$

Нетрудно убедиться, что множество $\left\{I_{k}: k \notin \Omega_{1}(x)\right\}$ (соответственно множество $\left\{\Delta_{j}\right.$ : $\left.\left.j \notin \Omega_{1}(y)\right\}\right)$ состоит из тех интервалов $I_{k}\left(\right.$ соответственно $\left.\Delta_{j}\right)$, которые пересекаются с одним из интервалов $d_{n}^{2 i}$; при этом с каждым из интервалов $d_{n}^{2 i}$ пересекаются не более пяти интервалов (так как $\left|I_{k}\right|=h>\varepsilon_{n+1}=\left|d_{n+1}^{2 l}\right|$, то по два интервала $I_{k}$ могут пересекаться с $d_{n+1}^{8 i-2}$ и $d_{n+1}^{8 i}$ и один может содержать внутри себя левьй конец интервала $d_{n}^{2 i}$, т.е. точку $t_{n}^{2 i-1}$ ). Учитьвая также, что в множество $\Omega_{3}(x)$ (соответственно множество $\left.\Omega_{3}(y)\right)$ входят те интервалы, которые пересекаются с ближайшим к $x$ (соответственно к $y$ ) интервалом $d_{n}^{2 i}$, будем иметь, что

$$
\# \Omega_{2}(x) \leqslant 5 \cdot 4^{n}, \quad \# \Omega_{2}(y) \leqslant 5 \cdot 4^{n}, \quad \# \Omega_{3}(x) \leqslant 5, \quad \# \Omega_{3}(y) \leqslant 5 .
$$

Для оценки величины $\Lambda V_{h}^{1,1}(F, x, y)$ разобьем ее на суммы следуюшим образом:

$$
\begin{aligned}
& \Lambda V_{h}^{1,1}(F, x, y)=\sum_{k \in \Omega_{1}(x), j \in \Omega(h)}+\sum_{k \in \Omega_{2}(x) \cup \Omega_{3}(x), j \in \Omega_{1}(y)}+\sum_{k \in \Omega_{2}(x), j \in \Omega_{2}(x) \cup \Omega_{3}(y)} \\
& +\sum_{k \in \Omega_{3}(x), j \in \Omega_{2}(y)}+\sum_{k \in \Omega_{3}(x), j \in \Omega_{3}(y)}=: A_{1}+A_{2}+A_{3}+A_{4}+A_{5} .
\end{aligned}
$$

Из (2.19), (2.23)-(2.25) получим

$$
A_{1}+A_{2} \leqslant C \omega\left(\delta_{n}\right)\left(\# \Omega_{1}(h)\right)(\# \Omega(h)) \leqslant C \omega\left(\delta_{n}\right) \frac{1}{\varepsilon_{n+1}^{2}} \leqslant C 4^{-n} .
$$

Далее, в силу (2.23) будем иметь, что

$$
k>\alpha(n) \quad \text { при } k \in \Omega_{2}(x), \quad j>\alpha(n) \quad \text { при } j \in \Omega_{2}(y) .
$$


Следовательно,

$$
\begin{aligned}
A_{3} & \leqslant C \omega\left(2^{-n}\right) \frac{1}{\lambda_{\alpha(n)}}\left(\# \Omega_{2}(x)\right)\left(\#\left(\Omega_{2}(y) \cup \Omega_{3}(y)\right)\right. \\
& \leqslant C 16^{-n} \omega\left(2^{-n}\right) 16^{n} \leqslant C \omega\left(2^{-n}\right), \\
A_{4} & \leqslant 5 \omega\left(2^{-n}\right) \frac{1}{\lambda_{\alpha(n)}}\left(\# \Omega_{2}(y)\right) \leqslant C \omega\left(2^{-n}\right) 16^{-n} 4^{n} \leqslant C \omega\left(\left(2^{-n}\right)\right) .
\end{aligned}
$$

Наконец, в силу $(2.24)$ и $(2.25)$ имеем $A_{5} \leqslant 25 \omega\left(2^{-n}\right)$. Из этих оценок находим, что

$$
\Lambda V_{h}^{1,1}(F, x, y) \leqslant C\left(4^{-n}+\omega\left(2^{-n}\right)\right), \quad \text { если } \varepsilon_{n+1}<h \leqslant \varepsilon_{n} .
$$

Оценивая аналогичным образом остальные два слагаемых в правой части (1.9), получим, что

$$
\Lambda V_{h}(F) \leqslant C\left(4^{-n}+\omega\left(2^{-n}\right)\right), \quad \text { если } \varepsilon_{n+1}<h \leqslant \varepsilon_{n} .
$$

Так как $n \rightarrow \infty$ при $h \rightarrow 0$, отсюда вытекает (2.22) (равномерно по $F$ вида (1.3)). Лемма 1 доказана.

Как было отмечено выше, теорема 1 вытекает непосредственно из теоремы 2 и леммы 1.

\section{СПИСОК ЦИТИРОВАННОЙ ЛИТЕРАТУРЫ}

[1] Бари Н. К. Тригонометрические ряды. М.: Физматгиз, 1961.

[2] Kahane J.-P., Katznelson Y. Séries de Fourier des fonctions bornées // Stud. Pure Math. à la Mémoire de P. Turan. Budapest: Acad. Kiado, 1983. P. 395-410.

[3] Саакян А. А. О свойствах коэффициентов Фурье суперпозиции функций // Докл. АН CCCP. 1979. T. 248. № 2. C. 302-306.

[4] Саакян А. А. О теореме Бора для кратных рядов Фурье // Матем. заметки. 1998. Т. 64. №6. C. 913-924.

[5] Арутюнян Ф. Г. Представление функций кратными рядами // Докл. АН АрмССР. 1977. T. 64 . № 2. C. $72-76$.

[6] Дьяченко М. И. Двумерные классы Ватермана и $u$-сходимость рядов Фурье // Матем. сб. 1999. Т. 190. № 7. С. 955-972.

[7] Голубов Б. И. Двойные ряды Фурье и функции ограниченной вариации // Изв. вузов. Сер. матем. 1972. № 12(127). С. 55-68.

[8] Саакян А.А. О сходимости двойных рядов Фурье функций ограниченной гармонической вариации // Изв. АН АрмССР. Сер. матем. 1986. Т. 21. №6. С. 517-529.

[9] Dyachenko M.I. Waterman classes and spherical partial sums of double Fourier series // Analysis Math. 1995. V. 21. № 1. P. 3-21.

[10] Бахвалов А. Н. Классы Ватермана и треугольные частичные суммы двойньх рядов Фурье // Analysis Math. 2001. T. 27. № 1. C. 3-36.

Институт математики НАН Армении

Поступило

E-mail: sart@instmath.sci.am

08.04 .2002

Исправленный вариант

17.10 .2002 